\title{
A STEADY-STATE CAPTURING METHOD FOR HYPERBOLIC SYSTEMS WITH GEOMETRICAL SOURCE TERMS*
}

\begin{abstract}
SHI JIN ${ }^{1}$
Abstract. We propose a simple numerical method for capturing the steady state solution of hyperbolic systems with geometrical source terms. We use the interface value, rather than the cell-averages, for the source terms that balance the nonlinear convection at the cell interface, allowing the numerical capturing of the steady state with a formal high order accuracy. This method applies to Godunov or Roe type upwind methods but requires no modification of the Riemann solver. Numerical experiments on scalar conservation laws and the one dimensional shallow water equations show much better resolution of the steady state than the conventional method, with almost no new numerical complexity.
\end{abstract}

Mathematics Subject Classification. 35L65, 65M06, 76B15.

Received: December 1st, 2000. Revised: February 7, 2001.

\section{INTRODUCTION}

Hyperbolic systems with geometric source terms arise in various applications ranging from shallow water with bottom tomography to nozzle flows. In such applications the source terms may have low regularity or possibly concentrations, which cause numerical difficulty when one wants to obtain the steady state solutions [9]. The numerical viscosity, an inevitable feature in modern shock capturing schemes, is blamed for the discrepancy of the steady state solutions.

In recent years there have been increasing interests in developing shock capturing numerical methods for such problems that are able to capture the steady state solution with better accuracy. A successful way to do this is to modify the numerical flux so that it takes into account the balance between the nonlinear flux and the source terms. This can be done by appropriately modifying the Godunov solvers, which leads to the so-called well-balanced schemes, introduced by Greenberg and Le Roux [9] and studied and further extended in $[3,4,6-8,10]$. LeVeque also proposed a modification of the Riemann solver which gives a scheme capable of capturing the quasi-steady wave propagation [15]. A kinetic scheme, with convergence proof for the scalar problem, was introduced by Botchorishvili et al. [2]. Another approach, which does not modify the Godunov solver, but approximating the source term in the upwind fashion using local characteristic decompositions, was introduced by Bernudez and Vazquez $[1,18]$. This was a nonlinear extension of an early idea of Roe for linear scalar equations [17]. All these methods capture the steady state solutions at cell averages either exactly or approximately with a formally second order accuracy.

\footnotetext{
Keywords and phrases. Hyperbolic systems, source terms, steady state solution, shallow water equations, shock capturing methods.

* Research supported in part by NSF grant No. DMS-9704957 and DMS-0196106.

1 Department of Mathematics, University of Wisconsin, Madison, WI 53706, USA. e-mail: jin@math.wisc.edu
} 
In this paper we propose a very simple method to handle this numerical difficulty. The designing principle for our method is to design numerical schemes that preserving the steady state at the cell interface. This philosophy clearly differs from all previous approaches where the schemes were designed to preserve the steady state at the cell-averages. The designing principle of our method, as a consequence, also allows us to obtain the cell-average steady state with a formally high order accuracy, which is easy to see for linear problems (as in Roe [17]) and was supported by strong numerical evidence for nonlinear problems in this paper. The change of the designing principle makes the design of the numerical methods strikingly simple. Both our method and the one of Bernudez and Vazquez (as well as some of the other schemes mentioned earlier) reduces to the method of Roe [17] for linear scalar problem. However, the method of [1] requires the local characteristic decomposition for the source terms, while our method uses the flux, which is already computed for the convection, directly in the source terms. Thus our method requires almost zero additional complexity on top of a standard shock capturing method for the homogeneous hyperbolic systems.

Our method applies to any shock capturing method in which the conserved variables (height of the water for the shallow water equations for example) at the cell-interface are available. Any method of Godunov or Roe type [14], in which the conserved variables at the cell-interface are available from the Riemann or approximate Riemann solver, belongs to this class.

In the sequel, we let $x_{j+1 / 2}$ be a grid point, $\left.x_{j}=\left(x_{j+1 / 2}+x_{j-1 / 2}\right) / 2\right)$ be the center of the cell $\left[x_{j-1 / 2}, x_{j+1 / 2}\right]$, $\Delta x=x_{j+1 / 2}-x_{j-1 / 2}$ be the uniform mesh size, and $t^{n}=n \Delta t$ be the discrete time with time step $\Delta t$. Let $u_{j}^{n}$ be the cell average of $u$ at $t^{n}$ over the cell $\left[x_{j-1 / 2}, x_{j+1 / 2}\right]$,

$$
u_{j}^{n}=\frac{1}{\Delta x} \int_{x_{j-1 / 2}}^{x_{j+1 / 2}} u\left(x, t^{n}\right) \mathrm{d} x
$$

and $u_{j+1 / 2}^{n}=u\left(x_{j+1 / 2}, t^{n}\right)$ be the approximate value of $u$ at the cell interface $x_{j+1 / 2}$.

We introduce the new method, referred to as the interface method, for the scalar problem in Section 2 and the shallow water equations with topography in Section 3. Formal second order accuracy at the steady state cell interface is shown. Numerical examples demonstrate the applicability and advantages of this method for both steady and unsteady solutions with regular or discontinuous bottom topographies.

\section{ONE-DIMENSIONAL SCALAR PROBLEM}

Consider the scalar conservation law with a source term

$$
\partial_{t} u+\partial_{x} f(u)=-z^{\prime}(x) b(u) .
$$

Assume

$$
a(u)=f^{\prime}(u), \quad D(u)=\int_{0}^{u} \frac{a(u)}{b(u)} \mathrm{d} s .
$$

The steady state solution is given by

$$
\partial_{x} f(u)+z^{\prime}(x) b(u)=0,
$$

which can be integrated out to give the algebraic expression

$$
D(u)+z(x) \equiv \text { constant } .
$$

A numerical scheme that preserves the steady state solution (2.4) exactly at the cell-averages requires

$$
D\left(u_{j}\right)+z\left(x_{j}\right)=\text { constant }, \quad \text { for all } j .
$$


A scheme that preserves the steady state solution (2.4) approximately at the cell-averages if

$$
D\left(u_{j}\right)+z\left(x_{j}\right)=\text { constant }+O\left((\Delta x)^{2}\right), \quad \text { for all } j .
$$

Due to the first order numerical viscosity used in shock capturing methods, a standard discretization (split or unsplit) over (2.1) can guarantee neither (2.5) nor (2.6). Rather, a first order numerical discretization error contributes to the numerical deviation from the steady state solution. This problem becomes more severe when the source terms lack regularity [2].

Past efforts have been focused on designing numerical methods that yield either (2.5) or (2.6) at steady state. By doing so one can obtain satisfactory results at the steady state.

\subsection{The interface method}

We propose a class of methods that is able to preserve the steady state solution (2.4) at the cell-interface, either exactly,

$$
D\left(u_{j+1 / 2}\right)+z_{j+1 / 2}=\text { constant }, \quad \text { for all } j,
$$

or approximately with a formally second order accuracy:

$$
D\left(u_{j+1 / 2}\right)+z_{j+1 / 2}=\text { constant }+O\left((\Delta x)^{2}\right), \quad \text { for all } j .
$$

Following this principle makes the designing of the numerical scheme extremely simple. In fact one only needs to use the interface value of $u$, which is already available in the numerical flux of the Godunov or Roe type, for the source term. Consequently, one also obtains a formally high order approximation to (2.4) not only at the cell interface but also for the cell averages. For linear scalar problems this is easy to see (as in Roe [17]). Although there is no theoretic support for this argument for nonlinear problems, our numerical evidence strongly supports this conclusion.

Bearing in mind (2.7) or (2.8), we propose the following method, which will be called the interface method thereafter, for (2.1):

$$
\partial_{t} u_{j}+\frac{f_{j+1 / 2}-f_{j-1 / 2}}{\Delta x}=-\frac{z_{j+1 / 2}-z_{j-1 / 2}}{\Delta x} \frac{b_{j+1 / 2}+b_{j-1 / 2}}{2},
$$

where $b_{j+1 / 2}=b\left(u_{j+1 / 2}\right)$. Here $f_{j+1 / 2}$ is the numerical flux of $f(u)$ defined at the cell interface $x_{j+1 / 2}$, and different definitions of the flux yield different shock capturing methods. For example, in the Godunov [5] or Roe [16] method, $f_{j+1 / 2}=f\left(u_{j+1 / 2}\right)$ where $u_{j+1 / 2}$ is the solution of the Riemann or approximate Riemann problem for $u_{t}+f(u)_{x}=0$ with initial data $u_{j}$ for $x<0$ and $u_{j+1}$ for $x>0$.

The interface method (2.9) provides a second order discretization of the source term. Although for a generic flux $f(u)$ it is impossible to derive a explicit form for $D(u)$, thus to verify $(2.7)$ or $(2.8)$, the numerical examples that follow and its extension to the shallow water problems in the next section show that either (2.7) or (2.8) is satisfied for smooth solutions.

The time marching method may be the standard forward Euler method or a second order Runge-Kutta method.

For numerical comparison we will also use the conventional method, where the source term is defined by the cell average of $u$ :

$$
\partial_{t} u_{j}+\frac{f_{j+1 / 2}-f_{j-1 / 2}}{\Delta x}=-\frac{z_{j+1 / 2}-z_{j-1 / 2}}{\Delta x} b\left(u_{j}\right) .
$$

This is referred to as the cell-average method. 
Remarks. If $D(u)$ is monotone, the following method for (2.1) is more accurate:

$$
\partial_{t} u_{j}+\frac{f_{j+1 / 2}-f_{j-1 / 2}}{\Delta x}=-\frac{z_{j+1 / 2}-z_{j-1 / 2}}{\Delta x} \frac{f_{j+1 / 2}-f_{j-1 / 2}}{D_{j+1 / 2}-D_{j-1 / 2}}
$$

The discretization of the source term is clearly second order.

For steady state solution, one drops the time derivative so (2.11) becomes

$$
\frac{f_{j+1 / 2}-f_{j-1 / 2}}{\Delta x}+\frac{z_{j+1 / 2}-z_{j-1 / 2}}{\Delta x} \frac{f_{j+1 / 2}-f_{j-1 / 2}}{D_{j+1 / 2}-D_{j-1 / 2}}=0,
$$

or simply

$$
D_{j+1 / 2}-D_{j-1 / 2}+z_{j+1 / 2}-z_{j-1 / 2}=0 .
$$

Thus one gets (2.6), so the correct steady state solution (2.4) is preserved exactly at the cell interface $x_{j+1 / 2}$.

\subsection{Numerical examples}

In this subsection we test the performance of the interface method (2.9) with several numerical examples. We use the Roe scheme with entropy fix for the numerical flux [15]:

$$
U_{j+1 / 2}^{n}=\left\{\begin{array}{lll}
U_{j}^{n}, & \text { if } & U_{j}^{n}+U_{j+1}^{n} \geq 0 \\
U_{j+1}^{n}, & \text { if } & U_{j}^{n}+U_{j+1}^{n}<0 \\
0, & \text { if } & U_{j}^{n}<0, U_{j+1}^{n}>0
\end{array}\right.
$$

The time discretization is the forward Euler method.

Example 2.1 We first use the Burgers equation with source term describing bathimetry in the SVS model:

$$
\begin{gathered}
\partial_{t} u+\partial_{x} \frac{u^{2}}{2}+z^{\prime}(x) u=0, \quad 0<x<10 \\
u(x, 0)=0 \quad \text { for } x>0, \quad u(0, t)=2 \text { for } t>0 .
\end{gathered}
$$

The steady state solution of this problem is given by the simple algebraic expression

$$
u+z=2 .
$$

For a continuous bottom the function $z(x)$ is chosen as

$$
z(x)= \begin{cases}\cos (\pi x), & 4.5 \leq x \leq 5.5 \\ 0, & \text { otherwise }\end{cases}
$$

For a discontinuous bottom we choose

$$
z(x)= \begin{cases}\cos (\pi x), & 5 \leq x \leq 6 \\ 0, & \text { otherwise }\end{cases}
$$

These are the examples used in [2]. Note that for $f(u)=u^{2} / 2$, method (2.12) is identical to method (2.9), thus the steady state solution is preserved exactly at the interface, as in (2.7). 

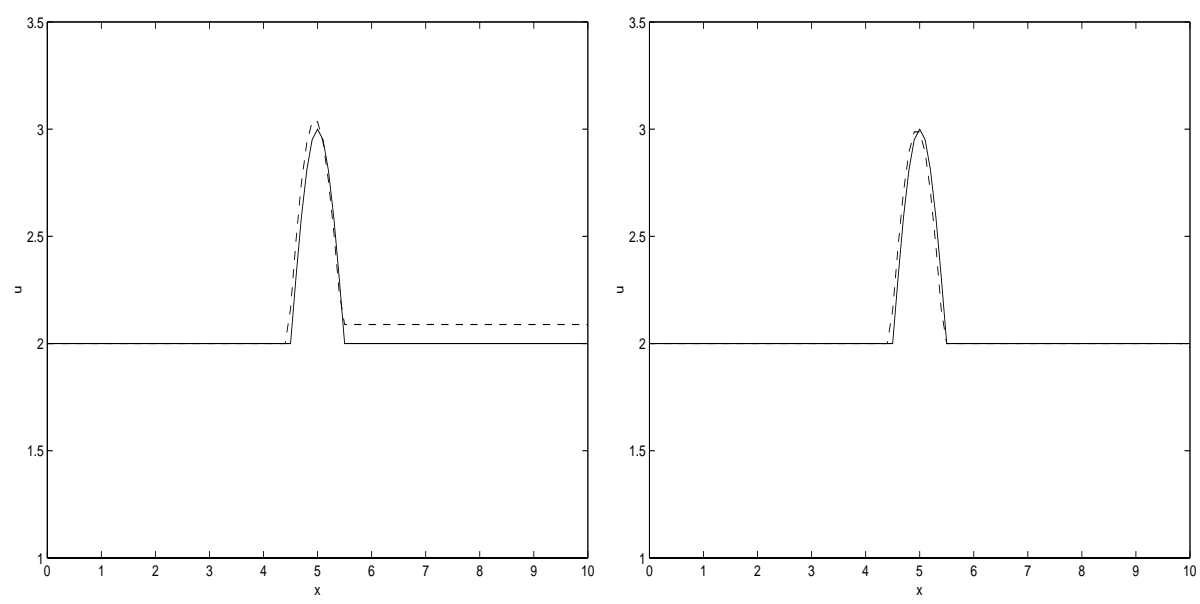

FiguRE 1. Numerical cell average solutions of $h+B$ compared with the exact solution at steady state for a continuous bottom. Solid line: the exact solution; dashed line: the numerical solution. Left: the cell-average method (2.10); right: the interface method (2.9).

TABLE 1. Comparison of errors for the continuous bottom case. Here "interface" stands for the interface method, while "average" is for the cell-average method.

\begin{tabular}{ccccc}
\hline $\begin{array}{c}\text { Number of } \\
\text { nodes }\end{array}$ & $\begin{array}{c}\text { Interface } \\
L^{\infty} \text { error }\end{array}$ & $\begin{array}{c}\text { Interface } \\
L^{1} \text {-error }\end{array}$ & $\begin{array}{c}\text { Average } \\
L^{\infty} \text { error }\end{array}$ & $\begin{array}{c}\text { Average } \\
L^{1} \text {-error }\end{array}$ \\
\hline 101 & $1.564 \times 10^{-1}$ & $1.000 \times 10^{-1}$ & $1.710 \times 10^{-1}$ & $5.025 \times 10^{-1}$ \\
201 & $7.846 \times 10^{-2}$ & $5.000 \times 10^{-2}$ & $8.594 \times 10^{-2}$ & $2.641 \times 10^{-1}$ \\
401 & $3.926 \times 10^{-2}$ & $2.500 \times 10^{-2}$ & $4.296 \times 10^{-2}$ & $1.354 \times 10^{-1}$ \\
801 & $1.963 \times 10^{-2}$ & $1.250 \times 10^{-2}$ & $2.150 \times 10^{-2}$ & $6.851 \times 10^{-2}$ \\
\hline
\end{tabular}

We use 100 grid points and time step $\Delta t=0.025$. For the continuous bottom, the cell average value of the exact steady state solution $(u=2-z)$ is compared with the numerical cell average by the cell-average method (2.10) in Figure 1 (left), while the comparison with the cell average of the numerical solution by the interface method (2.9) is shown in Figure 1 (right). One can see the numerical error beyond the bump for the cell-average method does not appear in the interface method. The $L^{1}$ and $L^{\infty}$ errors of these two methods for the steady state solution are given in Table 1 . We did not normalize the length of the domain (which is 10) in the calculation of the $L^{1}$ norm. Note that both methods converge linearly, in $L^{\infty}$ and $L^{1}$ norms, to the exact solution, and the $L^{\infty}$ errors (which is probably not the best measurement for a non-smooth solution) are of comparable size, but the $L^{1}$ errors of the interface method are about four times smaller.

Similar comparisons are made for the case of the discontinuous bottom, and the corresponding results are displayed in Figure 2. The difference between the interface and the cell-average methods is more striking for this case. As shown by Table 2, the $L^{1}$ error of the cell-average method (2.10) does not decay with shrinking grid sizes, while the interface method (2.9) converges linearly to the steady state and the errors are about the same size as in the case of continuous bottom. We do not compare the $L^{\infty}$ error since it is not a good measure for discontinuous solutions. 

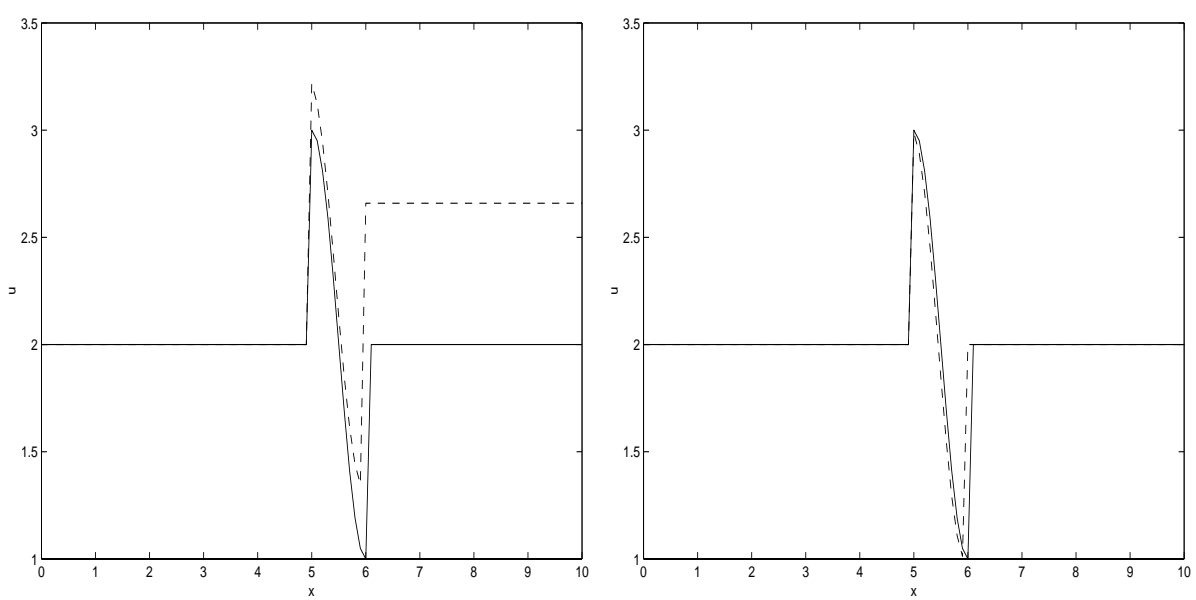

Figure 2. Numerical cell average solutions of $h+B$ compared with the exact solution at steady state for a discontinuous bottom. Solid line: the exact solution; dashed line: the numerical solution. Left: the cell-average method (2.10); right: the interface method (2.9).

TABLE 2. Comparison of errors for the discontinuous bottom case. Here "interface" stands for the interface method, while "average" is for the cell-average method.

\begin{tabular}{ccc}
\hline $\begin{array}{c}\text { Number of } \\
\text { nodes }\end{array}$ & $\begin{array}{c}\text { Interface } \\
L^{1} \text {-error }\end{array}$ & $\begin{array}{c}\text { Average } \\
L^{1} \text {-error }\end{array}$ \\
\hline 101 & $2.000 \times 10^{-1}$ & 2.983 \\
201 & $1.000 \times 10^{-1}$ & 2.811 \\
401 & $5.000 \times 10^{-2}$ & 2.769 \\
801 & $2.500 \times 10^{-2}$ & 2.654 \\
\hline
\end{tabular}

Example 2.2. Computation of the roll waves. Consider a model equation for the roll-wave:

$$
\begin{gathered}
\partial_{t} u+\partial_{x}\left(u^{2} / 2\right)=u \\
u(x, 0)=0.01 \sin (8 \pi x), \quad 0<x<1,
\end{gathered}
$$

This model is the weakly nonlinear asymptotic approximation of the shallow water equations with bottom and frictions, when the Froude number is over 2 [11,13]. Roll wave is a series of periodic traveling waves connected by shocks, and the solution of (2.20), starting with a zero mass, periodic initial data will tend to the steady state roll wave [12]. The problem (2.20) is linearly unstable due to the exponential growing mode from the source term, which inherits the physical instability of the roll wave in shallow water. This instability can be easily seen by integrating the equation over a periodic domain to get

$$
M^{\prime}(t)=\int u_{t}(x, t) \mathrm{d} x=\int_{-\infty}^{\infty} u(x, t) \mathrm{d} x=M(t),
$$

and hence,

$$
M(t)=M(0) \mathrm{e}^{t} .
$$

Hence the only solution that will remain bounded for all time is the one with zero mass, namely,

$$
M(t)=M(0)=0 .
$$


However, if a small perturbation to the initial data makes (2.15) zero mass condition violated, then the solution will grow exponentially. This instability contributes to the great numerical challenge for the roll wave simulation.

Let us consider the evolution of the total mass

$$
M^{n}=\Sigma_{j} U_{j}^{n}
$$

in the cell-average method

$$
u_{j}^{n+1}=u_{j}^{n}-\frac{\Delta t}{2 \Delta x}\left(\left(u_{j+1 / 2}^{n}\right)^{2}-\left(u_{j-1 / 2}^{n}\right)^{2}\right)+\Delta t u_{j}^{n}
$$

Summing over $j$ on (2.24) one gets

$$
M^{n}=(1+\Delta t) M^{n-1}=(1+\Delta t)^{n} M^{0} .
$$

So if the initial total mass $M^{0}$ of the numerical solution is not identically zero, the mass $M^{n}$ will grow algebraically and eventually becomes unbounded. Thus the success of a method for this problem depends on whether the method preserves the zero mass.

However, due to the finite machine precision, the initial mass $M^{0}$, though being zero analytically, is not exactly zero numerically. Rather it is a non-zero number in the order of machine precision. As a result of (2.25), this initial round-off error will be amplified by a factor of $1+\Delta t$ after each time step. Consequently, the solution of the cell-average method eventually becomes unbounded and can never converge to the steady state solution. Such a numerical instability is well-documented in [12].

The interface method,

$$
u_{j}^{n+1}=u_{j}^{n}-\frac{\Delta t}{2 \Delta x}\left(\left(u_{j+1 / 2}^{n}\right)^{2}-\left(u_{j-1 / 2}^{n}\right)^{2}\right)+\frac{\Delta t}{2}\left(u_{j+1 / 2}^{n}+u_{j-1 / 2}^{n}\right),
$$

although not always, does preserve the zero mass condition at the discrete level when the symmetry of the solution is preserved. Starting with the initial data for cell-average of $u$

$$
u_{i}^{0}=\frac{0.01}{8 \pi \Delta x}\left[\cos 8 \pi\left(x_{i}+\frac{\Delta x}{2}\right)-\cos 8 \pi\left(x_{i}-\frac{\Delta x}{2}\right)\right]
$$

and use one ghost cell on each side of the domain. Periodic condition with period 1 will be used to define the cell-average values at the ghost cells. All computations are made using double precision arithmetic. It is easy to check that, when one uses odd number of cells over each period of the sine wave, the Roe flux, given in (2.14), is perfectly symmetric with respect to the middle cell over one period, at which $u_{j}=0$, thus gives [12]

$$
M^{n}=M^{n-1} \equiv M^{0} .
$$

This key property is violated, however, if even number of cells is used over a period.

Since the initial data contains four sine wave lengths over the domain $[0,1]$, the exact solution should have four roll waves over $[0,1]$ with slope one, peak value 0.125 with jumps at $x=1 / 8,3 / 8,5 / 8$ and $7 / 8[12]$. In order to obtain the correct steady state, we need odd number of cells in each of the wave length. Namely, the zero mass condition should be preserved at every period of the sine wave. In this section we always use the interface method with the Godunov flux.

We use 204 cells over $[0,1]$, thus each period of the sine wave is resolved by 51 cells. We take $\Delta t=0.005$ and display the numerical solution between $0 \leq t \leq 30$ in Figure 3. One can see that in this case the exact solution has been captured and the steady state is already reached around $t=5$.

For more numerical examples for the roll wave simulations, see [12]. 


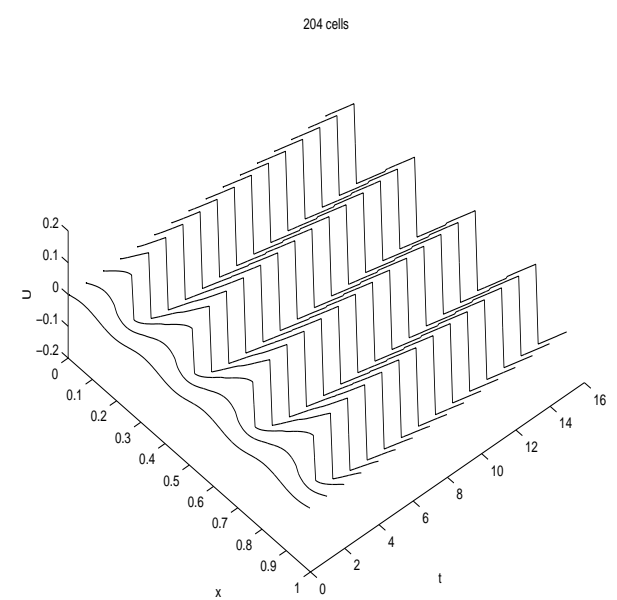

Figure 3. Evolution of roll waves, give by Example 2.2, by the interface method. There are 204 cells over $[0,1]$, so each period has 51 cells.

\section{Shallow WATER EQUATIONS With TOPOGRAPHY}

Consider the one-dimensional shallow water equations with topography:

$$
\begin{gathered}
\partial_{t} h+\partial_{x}(h u)=0, \\
\partial_{t}(h u)+\partial_{x}\left(h u^{2}+\frac{1}{2} g h^{2}\right)=-g h B^{\prime}(x) .
\end{gathered}
$$

Here $h$ is the depth of the water, $u$ is the mean velocity, $g$ is the gravitational constant, and $B(x)$ is the bottom elevation. This system describes flows in one-dimensional channel with the bottom elevation given by $B(x)$. The homogeneous shallow water equations are strictly hyperbolic with two distinct characteristic speeds $u \pm \sqrt{g h}$. The steady state solution of (2.1) is given by

$$
\begin{aligned}
\partial_{x}(h u) & =0, \\
\partial_{x}\left(h u^{2}+\frac{1}{2} g h^{2}\right) & =-g h B^{\prime}(x),
\end{aligned}
$$

or

$$
h u=\text { constant } \quad \text { and } \quad \frac{1}{2} u^{2}+g h+g B=\text { constant } .
$$

Our goal is to design a numerical approximation to (3.1-3.2) that captures this steady state solution with a formally high (at least second) order accuracy.

\subsection{The interface method}

We extend the interface method proposed for scalar problem into the shallow water equations (3.1-3.2). The scheme takes the following simple form:

$$
\begin{gathered}
\partial_{t} h_{j}+\frac{(h u)_{j+1 / 2}-(h u)_{j-1 / 2}}{\Delta x}=0 \\
\partial_{t}(h u)_{j}+\frac{\left(h u^{2}+\frac{1}{2} g h^{2}\right)_{j+1 / 2}-\left(h u^{2}+\frac{1}{2} g h^{2}\right)_{j-1 / 2}}{\Delta x}=-g \frac{h_{j+1 / 2}+h_{j-1 / 2}}{2} \frac{B_{j+1 / 2}-B_{j-1 / 2}}{\Delta x} .
\end{gathered}
$$


In (3.6), the flux is the Godunov or Roe type upwind flux using the Riemann or approximate Riemann solver [14]. The approximation of the source term is clearly second order. Next we analyze its accuracy at the steady state.

A special steady state solution, when the flow is at rest, is

$$
u \equiv 0, \quad h+B=\text { constant }
$$

It is easy to verify that the interface method (3.6-3.7) captures this solution exactly at the cell interface. Note that at the steady state, the method gives

$$
\begin{gathered}
\frac{(h u)_{j+1 / 2}-(h u)_{j-1 / 2}}{\Delta x}=0 \\
\frac{\left(h u^{2}+\frac{1}{2} g h^{2}\right)_{j+1 / 2}-\left(h u^{2}+\frac{1}{2} g h^{2}\right)_{j-1 / 2}}{\Delta x}=-g \frac{h_{j+1 / 2}+h_{j-1 / 2}}{2} \frac{B_{j+1 / 2}-B_{j-1 / 2}}{\Delta x} .
\end{gathered}
$$

If $u_{j+1 / 2} \equiv 0$ for all $j$, then (3.9) is automatically met, while (3.10) gives

$$
\frac{1}{2} g\left(h_{j+1 / 2}^{2}-h_{j-1 / 2}^{2}\right)=-\frac{1}{2} g\left(h_{j+1 / 2}+h_{j-1 / 2}\right)\left(B_{j+1 / 2}-B_{j-1 / 2}\right) .
$$

This implies that

$$
h_{j+1 / 2}+B_{j+1 / 2}=h_{j-1 / 2}+B_{j-1 / 2} \equiv \text { constant }
$$

Thus the steady state solution (3.8) is captured exactly at cell interfaces.

We now show that, even when the flow is not at rest, the interface method can still capture the steady state at cell interfaces with a formally second order accuracy. Assume $u \neq 0$. Equation (3.9) gives

$$
(h u)_{j+1 / 2}=C, \quad \text { or } \quad h_{j+1 / 2}=\frac{C}{u_{j+1 / 2}}
$$

where $C$ is a constant. Applying this in (3.10), one gets

$$
C\left(u_{j+1 / 2}-u_{j-1 / 2}\right)+\frac{1}{2} g\left(h_{j+1 / 2}^{2}-h_{j-1 / 2}^{2}\right)=-\frac{g}{2}\left(h_{j+1 / 2}+h_{j-1 / 2}\right)\left(B_{j+1 / 2}-B_{j-1 / 2}\right),
$$

or

$$
2 C \frac{u_{j+1.2}-u_{j-1 / 2}}{h_{j+1 / 2}+h_{j-1 / 2}}+g\left(h_{j+1 / 2}-h_{j-1 / 2}\right)=-g\left(B_{j+1 / 2}-B_{j-1 / 2}\right) .
$$

Using (3.13) in (3.15) one obtains

$$
2 \frac{\left(u_{j+1 / 2}-u_{j-1 / 2}\right) u_{j+1 / 2} u_{j-1 / 2}}{u_{j+1 / 2}+u_{j-1 / 2}}+g\left(h_{j+1 / 2}-h_{j-1 / 2}\right)=-g\left(B_{j+1 / 2}-B_{j-1 / 2}\right) .
$$

Since

$$
\begin{gathered}
2 \frac{\left(u_{j+1 / 2}-u_{j-1 / 2}\right) u_{j+1 / 2} u_{j-1 / 2}}{u_{j+1 / 2}+u_{j-1 / 2}}-\left(\frac{1}{2} u_{j+1 / 2}^{2}-\frac{1}{2} u_{j-1 / 2}^{2}\right) \\
=-\frac{1}{2} \frac{\left(u_{j+1 / 2}-u_{j-1 / 2}\right)^{3}}{u_{j+1 / 2}+u_{j-1 / 2}}=O\left((\Delta x)^{3}\right),
\end{gathered}
$$


equation (3.16) gives

$$
\left(\frac{1}{2} u_{j+1 / 2}^{2}-\frac{1}{2} u_{j-1 / 2}^{2}\right)+g\left(h_{j+1 / 2}-h_{j-1 / 2}\right)+g\left(B_{j+1 / 2}-B_{j-1 / 2}\right)=O\left((\Delta x)^{3}\right) .
$$

Thus for finite domain this implies

$$
\left(\frac{1}{2} u^{2}+g h+g B\right)_{j+1 / 2}=\text { constant }+O\left((\Delta x)^{2}\right), \quad \text { for any } \quad j
$$

Therefore the steady state solution (3.5) is captured at the cell interface with a second order accuracy, when the flow is not at rest.

To our knowledge this is the only method that has been shown to approximate the steady state solution (3.5) of the shallow water equations with a second order accuracy even when the flow is not at rest.

\subsection{Numerical examples}

In this section we present several numerical examples to evaluate the interface method for the shallow water equations. For comparison we introduce the cell-average method that uses the cell average of $h$ in the source term:

$$
\begin{aligned}
\partial_{t} h_{j}+\frac{(h u)_{j+1 / 2}-(h u)_{j-1 / 2}}{\Delta x} & =0, \\
\partial_{t}(h u)_{j}+\frac{\left(h u^{2}+\frac{1}{2} g h^{2}\right)_{j+1 / 2}-\left(h u^{2}+\frac{1}{2} g h^{2}\right)_{j-1 / 2}}{\Delta x} & =-g h_{j} \frac{B_{j+1 / 2}-B_{j-1 / 2}}{\Delta x} .
\end{aligned}
$$

In all the test below, the first or second order (with minmod slope limiters) Godunov flux for the convection and the corresponding value of $h$ at $x_{j+1 / 2}$ is used for the source term. The time discretization is the second order Runge-Kutta method.

Example 3.1. Flow at rest. In this example, the initial condition is a flow at rest. The channel is defined in domain $[0,25]$, with the bottom elevation

$$
B(x)= \begin{cases}-0.15 \times(x-10)^{2}+0.6, & 8<x<12 \\ 0, & \text { otherwise }\end{cases}
$$

The initial data are $h_{i}=1.5-\frac{1}{2}\left(B_{i-1 / 2}+B_{i+1 / 2}\right)$ and $u_{i} \equiv 0$. The zero-th order extrapolation is used at the boundary for ghost cells. The exact solution is $h+B=1.5$. We use 100 cells and $\Delta t=0.01$. The steady state cell-average solution of $h+B$, obtained by the interface method using first order Godunov flux, is depicted in Figure 4, along with $B(x)$. The numerical results at $t=20$, in which the cell-average of $h=B$, namely, the values of $h_{i}+\frac{1}{2}\left(B_{i-1 / 2}+B_{i+1 / 2}\right)$, are $8.3036 \times 10^{-4}$ in $L^{\infty}$ norm and $1.6988 \times 10^{-3}$ in $L^{1}$ norm within the exact value of 1.5 .

Example 3.2. Unsteady and steady state calculation with a non-zero velocity. In this example, we start with a Riemann initial data

$$
\begin{array}{lll}
h(x, 0)=1, & u(x, 0)=0, & \text { for } \quad x<0 \\
h(x, 0)=0.2, & u(x, 0)=0, & \text { for } \quad x>0 .
\end{array}
$$




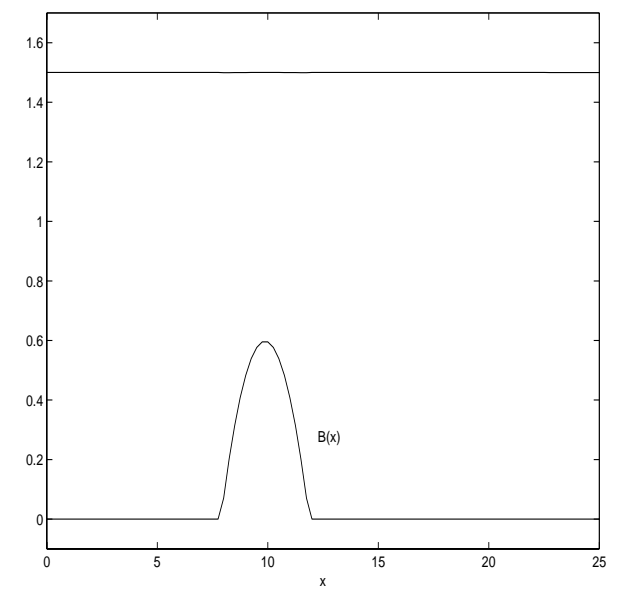

FiguRE 4. Numerical cell average solutions of $h+B$, obtained by the interface method using the first order Godunov flux, along with the bottom topography $B(x)$, at steady state for the shallow water equations. The flow velocity is zero.

The spatial domain is $[0,1]$. The boundary conditions are outflow conditions using the zeroth-order extrapolation. We use the second order Godunov flux for the convection and the source term. Without the source term, the solution to this Riemann problem consists of a right-moving shock and a left-moving rarefaction waves, as shown in Figure 5(a), which were computed with 100 cells and displayed at $t=0.25$. Consider the bottom function

$$
B(x)=1.398-0.347 \tanh (8 x-4)
$$

The numerical solution of the inhomogeneous problem, again using the same number of cells, are shown in Figure 5(b), along with the bottom function $B(x)$. The numerical solution is superimposed with the "exact" solution computed with 2000 cells (the solid line). This figure shows that the interface method is capable of computing unsteady solutions. Finally we display the steady state numerical solution, computed with 100 cells, in Figure 5(c). It can be seen that both $h u$ and $u^{2} / 2+h+B$ have reached the correct constants.

Example 3.3 A discontinuous bottom.

We solve the shallow water equation over domain $[0,1]$ with the bottom elevation

$$
B(x)= \begin{cases}0.35 \times[\cos (\pi(x-0.5) / 0.1)+1 .], & 0.3<x<0.5 \\ 0, & \text { otherwise }\end{cases}
$$

The initial data are $h_{i}=1-\frac{1}{2}\left(B_{i-1 / 2}+B_{i+1 / 2}\right)$ and $u_{i} \equiv 0$. The zero-th order extrapolation is used at the boundary for ghost cells. This is a subcritical flow with zero velocity. We will compare the performance between first and second order Godunov flux with the interface and the cell-average methods. We choose $\Delta x=0.01$ and $\Delta t=0.005$. The steady state cell-average solutions of $u^{2} / 2+h+B$ by both the interface and cell-average methods with the first order Godunov flux are depicted in Figure 6 (left), along with $B(x)$. The exact solution is $u^{2} / 2+h+B=1$. The discrepancy of the cell-average method appears in the domain where $B(x) \neq 0$, while in the interface method such an error is not visible by eye. The comparison for second order Godunov flux is made in Figure 6 (right), where the cell-average method gives more accurate result than its first order counterpart, but still gives result slightly inferior to that of the interface method. For the interface method the first and second order methods perform similarly at the steady state (except the kink across the discontinuity of $B(x)$. 


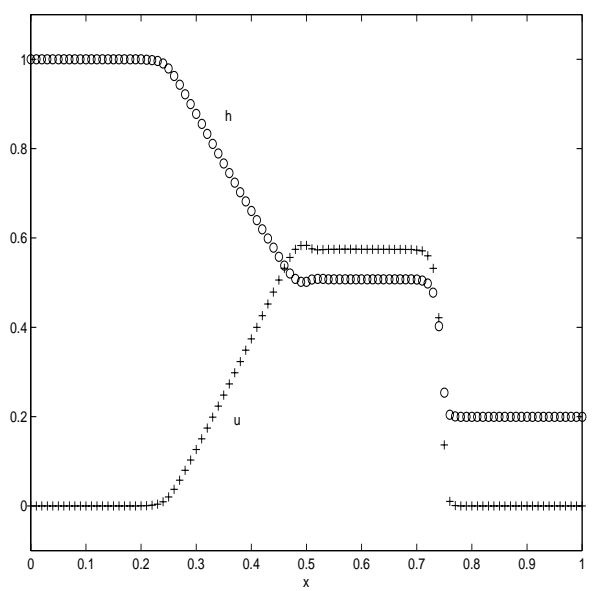

(a)

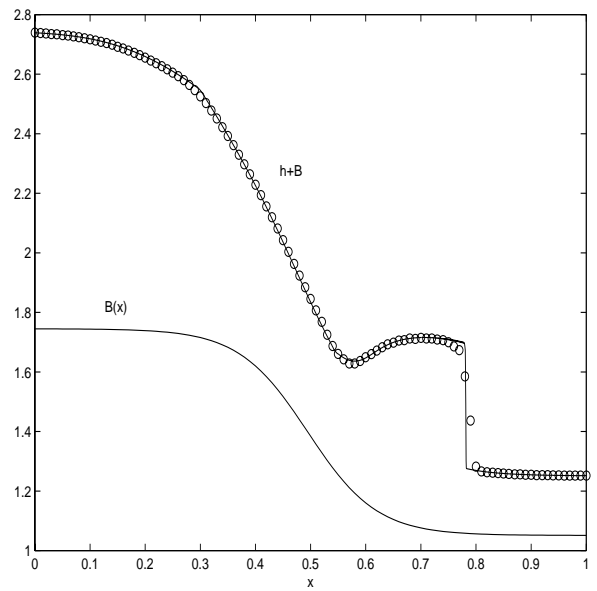

(b)

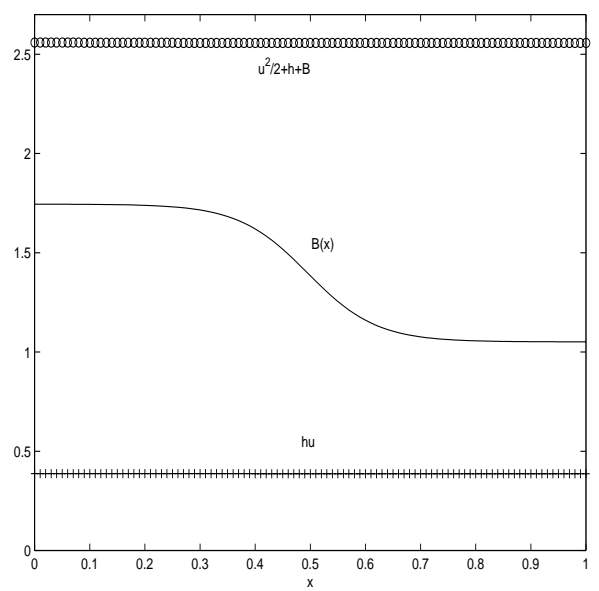

(c)

Figure 5. Numerical cell average solutions by the interface method for example 3.2. The velocity is nonzero. (a): Solution without the bottom at $t=0.25$; (b): Solution with bottom given in (3.26) at $t=0.25$; solid line: the exact solution; "o": the numerical solution. (c) Numerical cell average solutions along with the bottom topography $B(x)$ given in (3.26), at steady state.

Next, consider the bottom function

$$
B(x)= \begin{cases}0.35 \times[\cos (\pi(x-0.5) / 0.1)+1 .], & 0.4<x<0.5 ; \\ 0, \quad \text { otherwise } & \end{cases}
$$

we use the initial data are $h_{i}=1-\frac{1}{2}\left(B_{i-1 / 2}+B_{i+1 / 2}\right)$ and $u_{i} \equiv 0.1$. Here the flow is not at rest, but is still subcritical. The exact steady state solution is $u^{2} / 2+h+B=1.005$. The steady state solution of $u^{2} / 2+h+B$, where $u$ and $h$ are cell averages, for both the interface and cell-average methods with the first order Godunov flux are depicted in Figure 7 (left), along with $B(x)$. One can see that the interface method gives a better approximation than the cell-average method, although the result is slightly inferior to the zero-velocity case. 

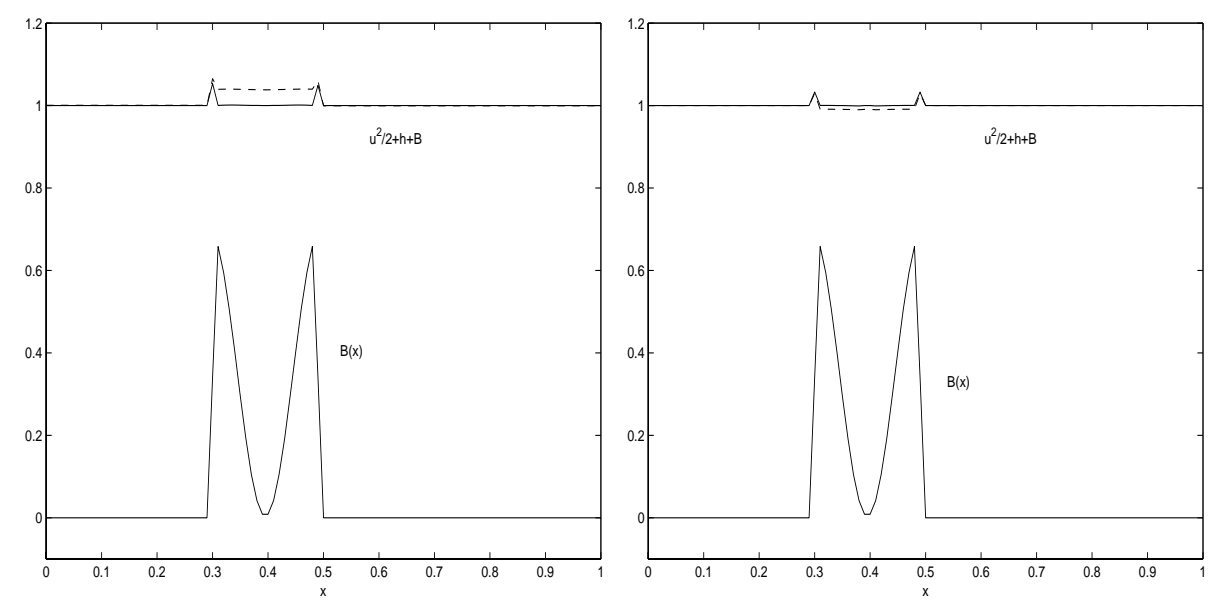

Figure 6. Flow at rest. Numerical cell average solutions of $u^{2} / 2+h+B$ at steady state. Solid line: the interface method; dashed line: the cell-average method. Left: The first order Godunov flux. Right The second order Godunov flux.
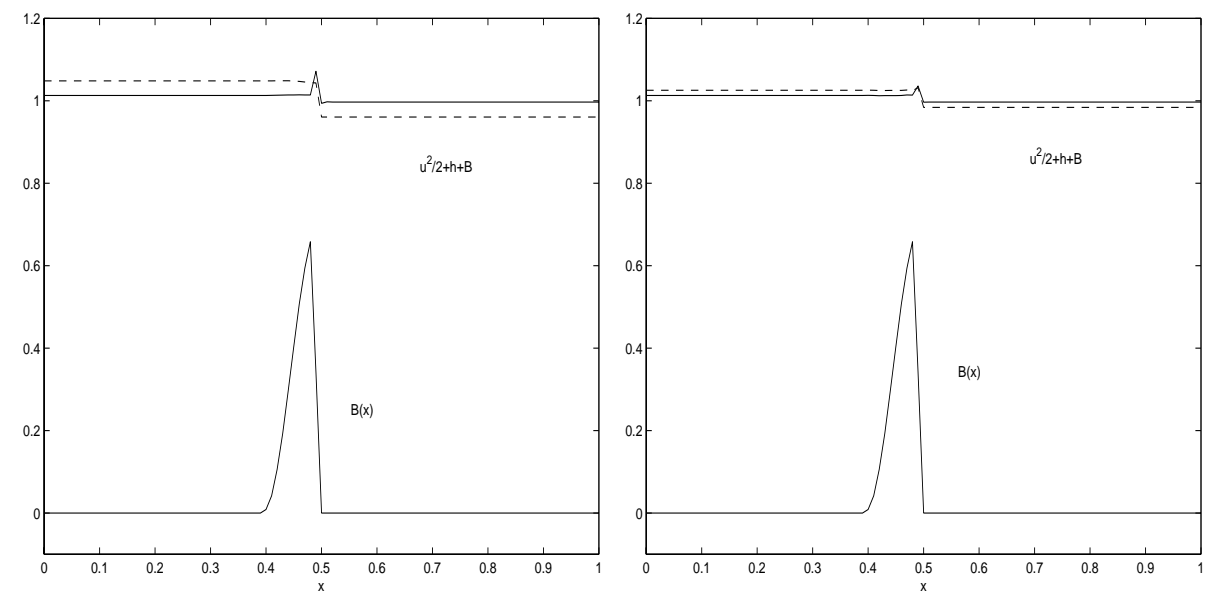

Figure 7. Flow at rest. Numerical cell average solutions of $u^{2} / 2+h+B$ at steady state. Solid line: the interface method; dashed line: the cell-average method. Left: The first order Godunov flux. Right: The second order Godunov flux.

Comparison of these two methods with the second order Godunov flux is made in Figure 7 (right). The cellaverage method performs better than the first order version, but is inferior compared to the interface method which improves slightly the result of its own first order version.

Example 3.4. Transcritical flow. This is an example from [15]. We solve the shallow water equations over domain $[0,1]$ with the bottom elevation

$$
B(x)=\left\{\begin{array}{l}
0.2 \times[\cos (\pi(x-0.5) / 0.1)+1 .], \quad 0.4<x<0.6 \\
0, \quad \text { otherwise }
\end{array}\right.
$$




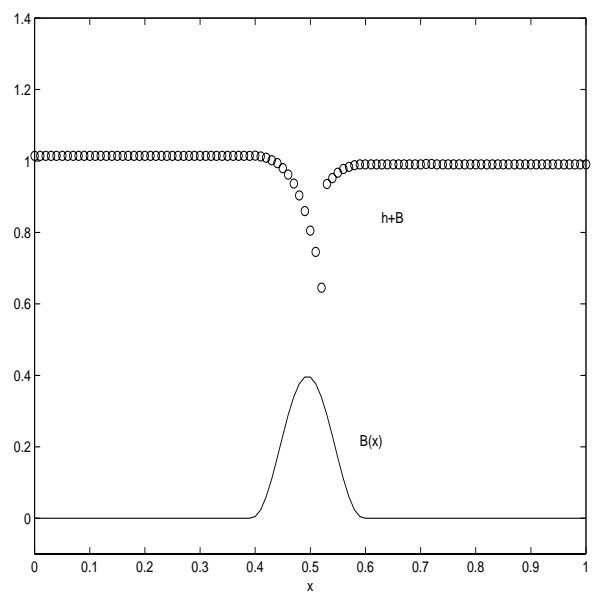

FiguRE 8. Numerical cell average solutions of $h+B$ at steady state for a transcritical flow over a ridge.

The initial data are $h_{i}=1-\frac{1}{2}\left(B_{i-1 / 2}+B_{i+1 / 2}\right)$ and $u_{i} \equiv 0.3$. The zero-th order extrapolation is used at the boundary for ghost cells. This is a transcritical flow, where the Froude number $F r=u / \sqrt{g h}$ passes over the critical value 1 near the peak of the bottom function $B(x)$ and the steady state solution contains a shock wave around that point. We use $\Delta x=0.01$ and $\Delta t=0.005$. The steady state result of the interface method using the second order Godunov flux is shown in Figure 8, where the numerical result is comparable to that of [15] and there is no oscillations. Numerical results for similar problems, as shown in [4], typically exhibit oscillations near the shock.

Acknowledgements. The author wishes to thank Prof. Benoît Perthame for many valuable discussions about this problem and the method proposed here.

\section{REFERENCES}

[1] A. Bernudez and M.E. Vazquez, Upwind methods for hyperbolic conservation laws with source terms. Comput. 83 Fluids 23 (1994) 1049-1071.

[2] R. Botchorishvili, B. Perthame and A. Vasseur, Equilibrium schemes for scalar conservation laws with stiff sources. Math. Comp. (to appear).

[3] A. Chinnayya and A.Y. Le Roux, A new general Riemann solver for the shallow-water equations with friction and topography. Preprint (1999).

[4] T. Gallouët, J.-M. Hérard and N. Seguin, Some approximate Godunov schemes to compute shallow-water equations with topography. AIAA J. (to appear 2001).

[5] S.K. Godunov, Finite difference schemes for numerical computation of solutions of the equations of fluid dynamics. Math. USSR-Sb. 47 (1959) 271-306.

[6] L. Gosse, A well-balanced flux-vector splitting scheme designed for hyperbolic systems of conservation laws with source terms. Comput. Math. Appl. 39 (2000) 135-159.

[7] L. Gosse, A well-balanced scheme using non-conservative products designed for hyperbolic systems of conservation laws with source terms. $M^{3} A S$ (to appear).

[8] L. Gosse and A.-Y. Le Roux, A well-balanced scheme designed for inhomogeneous scalar conservation laws. C. R. Acad. Sci. Paris Sér. I Math. 323 (1996). 543-546

[9] J.M. Greenberg and A.-Y. Le Roux, A well-balanced scheme for the numerical processing of source terms in hyperbolic equations. SIAM J. Numer. Anal. 33 1-16 1996.

[10] J.M. Greenberg, A.-Y. Le Roux, R. Baraille and A. Noussair, Analysis and approximation of conservation laws with source terms. SIAM J. Numer. Anal. 34 (1997) 1980-2007. 
[11] S. Jin and M. Katsoulakis, Hyperbolic systems with supercharacteristic relaxations and roll waves. SIAM J. Appl. Math. 61 (2000) 271-292 (electronic).

[12] S. Jin and Y.J. Kim, On the computation of roll waves. ESAIM: M2AN 35 (2001) 463-480.

[13] C. Kranenburg, On the evolution of roll waves. J. Fluid Mech. 245 (1992) 249-261.

[14] R.J. LeVeque, Numerical methods for conservation laws. Birkhäuser, Basel (1992).

[15] R.J. LeVeque, Balancing source terms and flux gradients in high-resolution Godunov methods: the quasi-steady wavepropagation algorithm. J. Comput. Phys. 146 (1998) 346-365.

[16] P.L. Roe, Approximate Riemann solvers, parameter vectors, and difference schemes. J. Comput. Phys. 43 (1981) 357-372.

[17] P.L. Roe, Upwind differenced schemes for hyperbolic conservation laws with source terms, in Nonlinear Hyperbolic Problems, Proc. Adv. Res. Workshop, St. Étienne, 1986, Lect. Notes Math. Springer, Berlin, 1270 (1987) 41-45.

[18] M.E. Vazquez-Cendon, Improved treatment of source terms in upwind schemes for shallow water equations in channels with irregular geometry. J. Comput. Phys. 148 (1999) 497-526.

To access this journal online: www.edpsciences.org 